HIP-2008-03/TH

1 February, 2008

revised 1 July, 2008

\title{
AN IMPROVED GLOBAL ANALYSIS OF NUCLEAR PARTON DISTRIBUTION FUNCTIONS INCLUDING RHIC DATA
}

\author{
Kari J. Eskola a,b,1, Hannu Paukkunena,b,2 and Carlos A. Salgadoc:3 \\ ${ }^{a}$ Department of Physics, P.O. Box 35, FI-40014 University of Jyväskylä, Finland \\ ${ }^{\mathrm{b}}$ Helsinki Institute of Physics, P.O. Box 64, FI-00014 University of Helsinki, Finland \\ ${ }^{\mathrm{c}}$ Departamento de Física de Partículas and IGFAE, Universidade de Santiago de \\ Compostela, Spain
}

\begin{abstract}
We present an improved leading-order global DGLAP analysis of nuclear parton distribution functions (nPDFs), supplementing the traditionally used data from deep inelastic lepton-nucleus scattering and Drell-Yan dilepton production in proton-nucleus collisions, with inclusive high- $p_{T}$ hadron production data measured at RHIC in $\mathrm{d}+\mathrm{Au}$ collisions. With the help of an extended definition of the $\chi^{2}$ function, we now can more efficiently exploit the constraints the different data sets offer, for gluon shadowing in particular, and account for the overall data normalization uncertainties during the automated $\chi^{2}$ minimization. The very good simultaneous fit to the nuclear hard process data used demonstrates the feasibility of a universal set of nPDFs, but also limitations become visible. The high- $p_{T}$ forward-rapidity hadron data of BRAHMS add a new crucial constraint into the analysis by offering a direct probe for the nuclear gluon distributions - a sector in the nPDFs which has traditionally been very badly constrained. We obtain a strikingly stronger gluon shadowing than what has been estimated in previous global analyses. The obtained nPDFs are released as a parametrization called EPS08.
\end{abstract}

\footnotetext{
${ }^{1}$ kari.eskola@phys.jyu.fi

${ }^{2}$ hannu.paukkunen@phys.jyu.fi

${ }^{3}$ carlos.salgado@cern.ch
} 


\section{Introduction}

With collider energies presently reached at BNL-RHIC, hard processes have become more and more important as diagnostic tools in the phenomenology of heavy ion collisions, QCD matter and QCD dynamics. The soon starting LHC heavy-ion program will emphasize the role of hard processes even further, by extending the kinematical range probed in the longitudinal momentum fraction $x$ and in the process virtuality scale $Q^{2}$ by several orders of magnitude with respect to the presently accessible ones. The existence of well-constrained up-to-date nuclear parton distribution functions (nPDFs) will thus be essential for the correct interpretation of the data.

Sets of collinearly factorized universal nPDFs, which are obtained in global perturbative QCD analyses paralleling those for the free proton, are available [1, 2, 13, 4, 5, 6, 7], see also Ref. [8]. These nPDFs are typically obtained by first parametrizing the nuclear corrections for each parton flavour relative to a known set of the free proton PDFs, and imposing constraints from sum rules at a chosen initial scale $Q_{0}^{2}$. A best fit to nuclear hard-process data from deep inelastic lepton-nucleus scattering (DIS) and the Drell-Yan (DY) process in proton-nucleus collisions is obtained by an iterative procedure which involves the DGLAP evolution [9] of the (absolute) nPDFs. The best global fit then fixes the initial nuclear corrections.

Since the first of the nPDF sets, EKS98 [1, 2], the procedure has been improved by performing the analysis at next-to-leading order (NLO) [6, 7] and by making uncertainty estimates [3, [5, 7] in analogy with the free proton case. In spite of such important progress, however, new data sets of relevance, which would more directly constrain the nuclear gluons in particular, have not been included in these ten years. The purpose of this paper is to make progress precisely in this respect, by including the data from inclusive high- $p_{T}$ hadron production in $\mathrm{d}+\mathrm{Au}$ collisions at RHIC in the global analysis of the nPDFs for the first time.

The most serious difficulty in the global DGLAP analyses of nPDFs has traditionally been the lack of experimental data which would impose stringent enough constraints for the nuclear gluon distributions. The extraction of the nPDFs would be cleanest in DIS but basically no high-precision data are at hand in the perturbative region $Q^{2} \gtrsim 1 \mathrm{GeV}^{2}$ at $x \lesssim 0.01$ there. This deficiency translates into a bad determination of the nPDFs, gluons in particular, in a region, where the nuclear effects are sizeable and which will be frequently accessed at the LHC. For the analysis of hard processes taking place at midrapidities at RHIC the situation has been better, as the available DIS and DY data constrain the nPDFs in most of the kinematic range probed. In the forward-rapidity domain, however, the hard processes are sensitive to nPDFs at smaller values of $x$ than what is presently constrained by DIS and DY: These RHIC data offer a possibility for independent further constraints of the nuclear gluon sector in the global analysis.

As discussed in previous works, [3, 10], the suppression in high- $p_{T}$ hadron production at forward rapidities in $\mathrm{d}+\mathrm{Au}$ collisions relative to $\mathrm{p}+\mathrm{p}$ collisions, measured by BRAHMS [11, would seem to suggest stronger gluon shadowing than, e.g., in the 
EKS98 set. This suppression has been also proposed as a signal of parton saturation being reached at RHIC [12], so that the compatibility of the measured suppression with DIS and DY data sets within the DGLAP framework is a question of special relevance from the QCD parton dynamics point of view as well.

In this paper, we shall demonstrate for the first time, that a global fit of a very good quality can indeed be obtained by simultaneously accommodating the DIS, DY and high- $p_{T}$ RHIC data in the leading-order (LO) DGLAP framework, i.e. that a relevant new set of universal, process-independent, collinearly factorized nPDFs can indeed be extracted, and that the gluon shadowing obtained at the smallest values of $x$ is indeed stronger than in previous global fits. Also limitations and uncertainties remaining in the analysis are discussed in light of the results obtained. An important new feature which we introduce in the global $\chi^{2}$-analysis here - borrowing it from the free proton analyses [13] - is the treatment of data normalization errors given by the RHIC experiments. In particular, we demonstrate that only by accounting for these systematic errors, a meaningful comparison with the RHIC high- $p_{T}$ pion data [14, 15, 16] can be done.

Parametrizing the obtained nuclear effects for each parton flavour in $x, Q^{2}$ and $A$ and making a simple fast computer code available for public use has proven to be a working idea in the past. Analogously with our previous EKS98 set [1, 2], we now release a new set of nPDFS called EPS08, which is available at [17].

The rest of this paper is organized as follows: In Sec. 2 we present the framework and analysis method, introducing the functional forms used and, in particular, the improvements in the $\chi^{2}$ fitting procedure. In Sec. 3 we present the results from the global fit, the new set of nPDFs, and the comparison with experimental data. The effects of the normalization-error treatment are demonstrated. In Sec. 4 we comment on the strong gluon shadowing solution found. Conclusions and outlook are presented in Sec. 5 .

\section{Framework and analysis method}

\subsection{Definition of nPDFs}

The DGLAP framework we use is essentially the same as in our previous global fits of nPDFs [1, 2, 3]. For each parton flavour $i$, we define the $\mathrm{nPDFs} f_{i}^{A}\left(x, Q^{2}\right)$ as the PDFs of protons bound to a nucleus of mass number $A$,

$$
f_{i}^{A}\left(x, Q^{2}\right) \equiv R_{i}^{A}\left(x, Q^{2}\right) f_{i}^{\mathrm{CTEQ} 6 \mathrm{~L} 1}\left(x, Q^{2}\right),
$$

where $f_{i}^{\text {CTEQ6L1 }}\left(x, Q^{2}\right)$ is obtained from the latest LO CTEQ set of the free proton PDFs [18], and $R_{i}^{A}\left(x, Q^{2}\right)$ is the nuclear modification factor for this parton flavour. We also assume that PDFs of bound neutrons can be obtained on the basis of isospin symmetry. For instance, the total $u$ quark $\mathrm{PDF}$ in a nucleus $A$ with $Z$ protons then becomes

$$
u_{A}\left(x, Q^{2}\right)=Z f_{u}^{A}\left(x, Q^{2}\right)+(A-Z) f_{d}^{A}\left(x, Q^{2}\right) .
$$


The nuclear effects of Deuterium $(A=2)$ and those in the cumulative region $x>1$ are neglected. We do not discuss the dependence of the nPDFs on transverse location inside the nucleus (the impact parameter dependence) [10, 19] here, either, only the average nuclear effects are considered.

We parametrize the nuclear modifications $R_{i}^{A}$ at an initial scale $Q_{0}^{2}=1.69 \mathrm{GeV}^{2}$, which conveniently matches the lowest scale and the charm-quark mass threshold in the CTEQ6L1 set. The valence quark modifications are constrained by baryon number conservation and the gluon modifications by momentum conservation. At higher scales $Q^{2}>Q_{0}^{2}$ the nPDFs are then obtained by solving the conventional DGLAP equations [9] at LO numerically, applying the fast solution method introduced in [20].

In order to reduce the amount of fitting parameters, and obtain a converging wellconstrained fit, we have to assume initially flavour-independent nuclear effects for valence quarks, sea quarks and gluons, i.e. we parametrize only three different functions: $R_{V}^{A}\left(x, Q_{0}^{2}\right)$ for all valence quarks, $R_{S}^{A}\left(x, Q_{0}^{2}\right)$ for all sea quarks, and $R_{G}^{A}\left(x, Q_{0}^{2}\right)$ for gluons. In the DGLAP evolution, each flavour is considered individually so that at $Q^{2}>Q_{0}^{2}$ the modifications may in principle depend on parton flavour.

\section{$2.2 \quad$ Fitting functions and parameters}

Choosing a suitable functional form for the input nuclear modification factors $R_{i}^{A}\left(x, Q_{0}^{2}\right)$ is among the most troublesome and crucial issues in the global nPDF analyses. On one hand the fit functions must be flexible enough, i.e. there should appear sufficiently many parameters so that all the relevant features suggested by the data can be caught. On the other hand, too large a number of parameters easily leads to badly converging fits. Thus the number of parameters always is a compromise between the flexibility of the fit and the feasibility of the $\chi^{2}$-analysis.

Up to now, gluon shadowing in the small- $x$ region $x \lesssim 10^{-2}$ has been constrained only by the momentum sum rule, and it has been essentially dictated by the assumed form of the fit function. Also the statistical error bars computed then reflect the uncertainties within the fit function chosen. Thus, as discussed in [3], there has been a large uncontrolled error in the gluon shadowing. The inclusion of the high- $p_{T}$ hadron data from RHIC at forward rapidities, however, now provides important further constraints for the gluon shadowing region, which will be exploited in the present work.

In order to take into account the RHIC high- $p_{T}$ forward-rapidity hadron data, which suggest a stronger gluon shadowing than obtained in previous global analyses (see the discussion in [3]), we need to modify the parametrization of the shadowing region. In our past works, we assumed a saturation of the modifications $R_{i}^{A}\left(x, Q_{0}^{2}\right)$ such that $R_{G}^{A} \approx R_{S}^{A} \rightarrow$ const at $x \rightarrow 0$. This assumption is relaxed in the present analysis, and we introduce a power-law behaviour of the nuclear modification factors at small $x$ as follows

$$
R_{i}^{A}\left(x, Q_{0}^{2}\right) \stackrel{x \rightarrow 0}{\sim} x^{\alpha^{A}}, \quad \alpha^{A}>0
$$

This can be motivated by the (approximate) power-law behaviour $\sim x^{-P_{\text {free }}}$ of the free proton PDFs at small- $x$, assuming that the $\mathrm{nPDF}$ s share this same gross feature but 
with a different power $P_{\text {free }} \geq P_{\text {bound }}$. This means that $R_{i}^{A} \sim x^{\left(P_{\text {free }}-P_{\text {bound }}\right)} \rightarrow 0$ as $x \rightarrow 0$.

With this assumption as a guide, we parametrize the initial nuclear modifications $R_{V}^{A}, R_{S}^{A}$ and $R_{G}^{A}$ in three pieces as illustrated in Fig. 1: $R_{1}^{A}(x)$ at small values of $x$, below the antishadowing maximum, $x \leq x_{a}^{A} ; R_{2}^{A}(x)$ from the antishadowing maximum to the EMC minimum, $x_{a}^{A} \leq x \leq x_{e}^{A}$; and $R_{3}^{A}(x)$ in the large- $x$ Fermi-motion region, $x \geq x_{e}^{A}$

$$
\begin{array}{ll}
R_{1}^{A}(x)=c_{0}^{A}+\left(c_{1}^{A}+c_{2}^{A} x^{\alpha^{A}}\right)\left[\exp \left(-x / x_{s}^{A}\right)-\exp \left(-x_{a}^{A} / x_{s}^{A}\right)\right], & x \leq x_{a}^{A} \\
R_{2}^{A}(x)=a_{0}^{A}+a_{1}^{A} x+a_{2}^{A} x^{2}+a_{3}^{A} x^{3}, & x_{a}^{A} \leq x \leq x_{e}^{A} \\
R_{3}^{A}(x)=\frac{b_{0}^{A}-b_{1}^{A} x}{(1-x)^{\beta^{A}}}+b_{2}^{A}\left(x-x_{e}\right)^{2}, & x_{e}^{A} \leq x \leq 1 .
\end{array}
$$

Some of the parameters above are eliminated by matching the different pieces smoothly together: we require continuity of the fit functions and zero first-derivatives at the antishadowing maximum $x_{a}^{A}$ and at the EMC minimum $x_{e}^{A}$. The required behavior (2) fixes $c_{0}^{A}$. It is convenient to express the fit functions in terms of the following 8 parameters,

$\alpha^{A} \quad$ the power according to which $R_{1}^{A} \rightarrow 0$ at $x \rightarrow 0$,

$x_{s}^{A} \quad$ a slope factor in the exponential,

$x_{a}^{A}, y_{a}^{A}$ position and height of the antishadowing maximum

$x_{e}^{A}, \quad$ position of the EMC minimum

$\Delta_{e}^{A} \quad$ difference of the antishadowing maximum and the EMC minimum

$\beta^{A}, b_{2}^{A}$ slope factors in the Fermi-motion part $R_{3}$ at $x>x_{e}$.

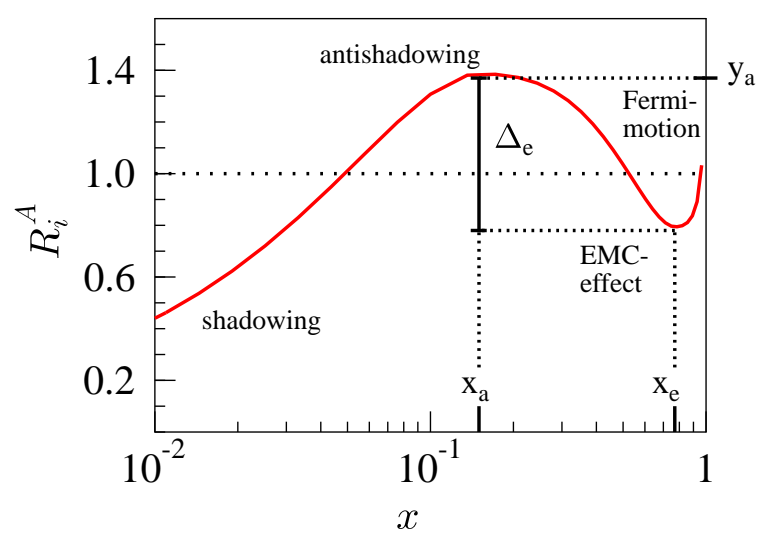

Figure 1: An illustration of the smoothly matched fit functions $R_{i}^{A}(x)$ and the role of the parameters $x_{a}^{A}, y_{a}^{A}, x_{e}^{A}$ and $\Delta_{e}^{A}$. The superscripts $A$ have been suppressed in the figure.

In principle, all parameters above are different from one nucleus to another, hence 
the superscript $A$. For the $A$ dependence we assume a simple power law,

$$
z_{i}^{A}=z_{i}^{A_{\mathrm{ref}}}\left(\frac{A}{A_{\mathrm{ref}}}\right)^{p_{z_{i}}}
$$

where $z_{i}=x_{s}, x_{a}, y_{a} \ldots$, and choose the reference nucleus to be Carbon, $A_{\text {ref }}=12$.

With momentum and baryon number sum rules, we can fix $\alpha^{A}$ for gluons and valence quarks, and thus reduce the total number of parameters to 44 . This is still far too many for a convergent $\chi^{2}$-minimization with the nuclear data constraints available. To proceed, additional assumptions need to be introduced - how the unconstrained regions of the phase space are handled, and how the number of final fit parameters is reduced down to 15 by fixing those parameters which cannot be constrained, is explained in Sec. 3.1 .

\subsection{Data sets}

The experimental data, providing the nonperturbative input for the nPDFs, in our present analysis covers three types of hard processes involving nuclei: In addition to the data from lepton-nucleus deep inelastic scattering and proton-nucleus Drell-Yan dilepton production, as a new ingredient we include the data from inclusive high- $p_{T}$ hadron production in minimum-bias $\mathrm{d}+\mathrm{Au}$ collisions from the BRAHMS, PHENIX and STAR collaborations at RHIC. In total, we have over 600 data points covering 13 nuclei from Helium up to Lead. Table 1 summarizes the data sets in our analysis.

The (minimum bias) DIS and DY data that we utilize are available as ratios of differential cross sections between a nucleus $A$ and a reference nucleus. We denote the cross section ratios computed against deuterium as

$$
R_{\mathrm{DIS}}^{\mathrm{A}}\left(x, Q^{2}\right) \equiv \frac{\frac{1}{A} d \sigma_{\mathrm{DIS}}^{l \mathrm{~A}} / d Q^{2} d x}{\frac{1}{2} d \sigma_{\mathrm{DIS}}^{l \mathrm{~d}} / d Q^{2} d x} \stackrel{\mathrm{LO}}{=} R_{F_{2}}^{\mathrm{A}}\left(x, Q^{2}\right), \quad R_{\mathrm{DY}}^{\mathrm{A}}\left(x, M^{2}\right) \equiv \frac{\frac{1}{A} d \sigma_{\mathrm{DY}}^{\mathrm{pA}} / d M^{2} d x}{\frac{1}{2} d \sigma_{\mathrm{DY}}^{\mathrm{pd}} / d M^{2} d x}
$$

where $x$ refers to the momentum fraction and $Q^{2}$ to the photon virtuality for DIS, and $M^{2}$ to the invariant mass of the lepton pair and $x$ to either $x_{1}$ or $x_{2}$ for DY. The scales $Q^{2}$ and $M^{2}$ also define our factorization scales, and we consider only those data points which lie above our initial scale: $M^{2}, Q^{2}>1.69 \mathrm{GeV}^{2}$.

The inclusive hadron production data at RHIC comes as the nuclear modification factor $R_{\mathrm{dAu}}$, the ratio between the invariant yields in $\mathrm{d}+\mathrm{Au}$ and $\mathrm{p}+\mathrm{p}$ collisions,

$$
R_{\mathrm{dAu}}=\frac{1}{\left\langle N_{\text {coll }}\right\rangle} \frac{d^{2} N^{\mathrm{dAu}} / d p_{T} d \eta}{d^{2} N^{\mathrm{pp}} / d p_{T} d \eta} \stackrel{\text { min.bias }}{=} \frac{\frac{1}{2 A} d^{2} \sigma^{\mathrm{dAu}} / d p_{T} d \eta}{d^{2} \sigma^{\mathrm{pp}} / d p_{T} d \eta}
$$

where $p_{T}$ and $\eta$ denote the transverse momentum and the pseudorapidity of the observed hadron, and $\left\langle N_{\text {coll }}\right\rangle$ is the estimated average number of inelastic binary nucleonnucleon collisions in a centrality class studied. The last equality holds for the minimum 


\begin{tabular}{|c|c|c|c|c|c|c|}
\hline Experiment & Process & Nuclei & Data points & $\chi^{2}$ & Weight & Ref. \\
\hline SLAC E-139 & DIS & $\overline{\mathrm{CHe}(4) / \mathrm{D}}$ & 18 & 2.0 & 1 & {$[25]$} \\
\hline NMC 95, reanalysis & DIS & $\mathrm{He} / \mathrm{D}$ & 16 & 12.1 & 1 & $26]$ \\
\hline NMC 95 & DIS & $\mathrm{Li}(6) / \mathrm{D}$ & 15 & 30.7 & 1 & 27 \\
\hline SLAC E-139 & DIS & $\operatorname{Be}(9) / \mathrm{D}$ & 17 & 5.5 & 1 & 25 \\
\hline NMC 96 & DIS & $\mathrm{Be}(9) / \mathrm{C}$ & 15 & 4.2 & 1 & 28 \\
\hline SLAC E-139 & DIS & $\mathrm{C}(12) / \mathrm{D}$ & 7 & 3.5 & 1 & 25 \\
\hline NMC 95 & DIS & $\mathrm{C} / \mathrm{D}$ & 15 & 10.5 & 5 & 27 \\
\hline NMC 95, reanalysis & DIS & $\mathrm{C} / \mathrm{D}$ & 16 & 17.8 & 5 & 26 \\
\hline NMC 95, reanalysis & DIS & $\mathrm{C} / \mathrm{Li}$ & 20 & 36.4 & 1 & 26 \\
\hline FNAL-E772 & DY & $\mathrm{C} / \mathrm{D}$ & 9 & 8.9 & 10 & 29 \\
\hline SLAC E-139 & DIS & $\mathrm{Al}(27) / \mathrm{D}$ & 17 & 3.6 & 1 & 25 \\
\hline NMC 96 & DIS & $\mathrm{Al} / \mathrm{C}$ & 15 & 6.7 & 1 & 28 \\
\hline SLAC E-139 & DIS & $\mathrm{Ca}(40) / \mathrm{D}$ & 7 & 1.3 & 1 & 25 \\
\hline FNAL-E772 & DY & $\mathrm{Ca} / \mathrm{D}$ & 9 & 5.0 & 10 & 29 \\
\hline NMC 95, reanalysis & DIS & $\mathrm{Ca} / \mathrm{D}$ & 15 & 27.9 & 1 & 26 \\
\hline NMC 95, reanalysis & DIS & $\mathrm{Ca} / \mathrm{Li}$ & 20 & 26.1 & 1 & $26]$ \\
\hline NMC 96 & DIS & $\mathrm{Ca} / \mathrm{C}$ & 15 & 6.3 & 1 & 28 \\
\hline SLAC E-139 & DIS & $\mathrm{Fe}(56) / \mathrm{D}$ & 23 & 16.5 & 1 & 25 \\
\hline FNAL-E772 & DY & $\mathrm{Fe} / \mathrm{D}$ & 9 & 5.0 & 10 & 29 \\
\hline NMC 96 & DIS & $\mathrm{Fe} / \mathrm{C}$ & 15 & 11.9 & 1 & 28 \\
\hline FNAL-E866 & DY & $\mathrm{Fe} / \mathrm{Be}$ & 28 & 21.6 & 1 & 30 \\
\hline CERN EMC & DIS & $\mathrm{Cu}(64) / \mathrm{D}$ & 19 & 12.3 & 1 & 31 \\
\hline SLAC E-139 & DIS & $\operatorname{Ag}(108) / D$ & 7 & 2.3 & 1 & 25 \\
\hline NMC 96 & DIS & $\operatorname{Sn}(117) / C$ & 15 & 10.9 & 1 & 28 \\
\hline NMC $96, Q^{2}$ dep. $x \leq 0.025$ & DIS & $\mathrm{Sn} / \mathrm{C}$ & 24 & 9.4 & 10 & 32 \\
\hline NMC $96, Q^{2}$ dep. $x>0.025$ & DIS & $\mathrm{Sn} / \mathrm{C}$ & 120 & 75.2 & 1 & 32 \\
\hline FNAL-E772 & DY & $\mathrm{W}(184) / \mathrm{D}$ & 9 & 10.0 & 10 & 29 \\
\hline FNAL-E866 & DY & $\mathrm{W} / \mathrm{Be}$ & 28 & 26.5 & 1 & 30 \\
\hline SLAC E-139 & DIS & $\mathrm{Au}(197) / \mathrm{D}$ & 18 & 6.1 & 1 & 25 \\
\hline RHIC-BRAHMS & $h^{-}$prod. & $\mathrm{dAu} / \mathrm{pp}$ & 6 & 2.2 & 40 & 11 \\
\hline RHIC-PHENIX & $\pi^{0}$ prod. & $\mathrm{dAu} / \mathrm{pp}$ & 35 & 21.3 & 1 & 14, 15 \\
\hline RHIC-STAR & $\pi^{+}+\pi^{-}$prod. & $\mathrm{dAu} / \mathrm{pp}$ & 10 & 3.5 & 1 & [16] \\
\hline NMC 96 & DIS & $\mathrm{Pb} / \mathrm{C}$ & 15 & 5.1 & 1 & 28 \\
\hline
\end{tabular}

Table 1: The data used in this analysis. The mass numbers are indicated in parentheses and the number of data points refers to those falling within our kinematical cuts, $Q^{2}, M^{2} \geq$ $1.69 \mathrm{GeV}^{2}$ for DIS and DY, and $p_{T} \geq 2 \mathrm{GeV}$ for hadron production at RHIC. The quoted $\chi^{2}$ values correspond to the unweighted contributions of each data set. 
bias case which we are interested in here. According to the QCD factorization theorem, the inclusive hadron production cross sections can be computed as

$$
d \sigma^{A B \rightarrow h+X}=\sum_{i j k l} f_{i}^{A}\left(Q^{2}\right) \otimes f_{j}^{B}\left(Q^{2}\right) \otimes \sigma^{i j \rightarrow k l}\left(Q^{2}\right) \otimes D_{k \rightarrow h+X}\left(Q_{f}^{2}\right),
$$

where $f_{i}^{A}\left(Q^{2}\right)$ are the input nPDFs, $\sigma^{i j \rightarrow k l}\left(Q^{2}\right)$ is the pQCD matrix element squared, and $D_{k \rightarrow h+X}\left(Q_{f}^{2}\right)$ denotes the fragmentation functions for which we use the KKP parametrization [21]. Our choice for the fragmentation scale is the hadronic transverse momentum, $Q_{f}^{2}=p_{T}^{2}$, and for the factorization and renormalization scale $Q^{2}$ we take the corresponding partonic transverse momentum. Notice here that all the scale choices above are simplifications in the sense that they could be left as additional fit parameters, and that the KKP fragmentation functions do not distinguish negatively and positively charged hadrons. Such details, however, are beyond the scope of the present analysis. A more detailed discussion of how the needed differential cross sections (7) are calculated in practice in LO can be found in 22 .

We should emphasize that the following choices are made with the inclusion of the RHIC data:

- Since we do not discuss the impact parameter dependent nuclear effects here, we consider only minimum bias data for $R_{\mathrm{dAu}}$.

- From PHENIX and STAR, we systematically include only the pion production data. This is because the 'Cronin-type' enhancement seems to be much larger for baryons than for mesons [14], signalling of the fact that still at $\sqrt{s}_{\mathrm{NN}}=200 \mathrm{GeV}$ there might be a significant component of nonperturbative baryon-number transport from the beam particles. We do, however, include the BRAHMS data for negatively charged hadrons with the assumption that the antiproton content in this data sample is negligible.

- Perturbative QCD calculations of inclusive hadron production, which are performed strictly in the framework of collinear factorization (without any intrinsic transverse momentum), show similar features both in LO [22] and in NLO [23, 24]: Below $p_{T} \sim$ a few $\mathrm{GeV}$ the computed cross sections for $p+p(\bar{p})$ collisions start to overshoot the data. Hence, since we should not push the nuclear case too far either, we choose to include only the region $p_{T} \geq 2 \mathrm{GeV}$ of the RHIC data on inclusive hadron production in this analysis. More discussion on this choice will follow later.

\subsection{Modified $\chi^{2}$}

The established way of fitting a set of parameters $\{z\}$ of the PDFs against a large number of experimental data, is the minimization of the global $\chi^{2}$ function. In its simplest form the global $\chi^{2}$, the goodness parameter of the fit obtained, is defined by

$$
\chi^{2}(\{z\}) \equiv \sum_{N} \chi_{N}^{2}(\{z\}),
$$


where $N$ labels the experimental data sets, and

$$
\chi_{N}^{2}(\{z\}) \equiv \sum_{i \in N}\left[\frac{D_{i}-T_{i}(\{z\})}{\sigma_{i}}\right]^{2},
$$

where $D_{i}, \sigma_{i}$, and $T_{i}(\{z\})$ denote the value of a single data point, its measurement uncertainty, and the corresponding theoretical value which depends on the parameters $\{z\}$ of PDFs.

For the nPDF analyses involving only DIS and DY data, the simple form of $\chi^{2}$ above has been sufficient, but for our current purposes a more general definition for the $\chi^{2}$, introduced in [13], is needed:

$$
\begin{aligned}
\chi^{2}(\{z\}) & \equiv \sum_{N} w_{N} \chi_{N}^{2}(\{z\}) \\
\chi_{N}^{2}(\{z\}) & \equiv\left(\frac{1-f_{N}}{\sigma_{N}^{\text {norm }}}\right)^{2}+\sum_{i \in N}\left[\frac{f_{N} D_{i}-T_{i}(\{z\})}{\sigma_{i}}\right]^{2},
\end{aligned}
$$

where $w_{N}$ is a weight factor chosen separately for each data set, $\sigma_{N}^{\text {norm }}$ is the relative uncertainty in the overall normalization reported by the experiment, and $f_{N}$ is the optimized value of the overall normalization for the data set, corresponding to each parameter set $\{z\}$. The reasons for the necessity of such redefinition are the following:

1. By making the weight factor $w_{N}$ larger than 1 , we can emphasize by hand the importance of those data sets which contain definite physics content - such as constraints for small- $x$ gluons - but whose number of data points is small. With a default value $w_{N}=1$, such data sets would have a negligible contribution to the overall $\chi^{2}$ and the valuable constraints they offer would escape unnoticed.

2. In addition to the point-to-point statistical and systematic errors, certain data sets have a significant common normalization uncertainty $\sigma_{N}^{\text {norm }}$ for all data points within the set. Even if this normalization uncertainty is large, the shape of the distribution formed by the data points may be a valuable constraint for the nPDFs. We introduce for each data set a normalization factor $f_{N} \in[1-$ $\left.\sigma_{N}^{\text {norm }}, 1+\sigma_{N}^{\text {norm }}\right]$ which multiplies all the experimental values within the set $N$. In connection with $f_{N}$, there is an additional "penalty" factor $\left(\frac{1-f_{N}}{\sigma_{N}^{\text {norm }}}\right)^{2}$ which is the larger the more $f_{N}$ deviates from unity - this accounts for the fact that having $f_{N}=1$ is anyway the experiment's best estimate for normalization. The actual value for $f_{N}$ is determined from the requirement that $\chi_{N}^{2}(\{z\})$ for each data set is at minimum.

The motivation for both modifications in the $\chi^{2}$ definition discussed above comes mainly from adding the RHIC data for the nuclear modification factor $R_{\mathrm{dAu}}$ of Eq. (6) into the analysis. First, the BRAHMS data set for forward direction $(\eta \sim 2 \ldots 3$, especially with our choice $p_{T} \geq 2 \mathrm{GeV}$ ) has only a very few data points. These would not 
have much effect in the global $\chi^{2}$ without being artificially emphasized. Second, the average number of inelastic binary nucleon-nucleon collisions $\left\langle N_{\text {coll }}\right\rangle$ in $\mathrm{d}+\mathrm{Au}$ collision is derived from a simulation of the experiment with the Glauber model as an input, which gives rise to a significant model-dependent normalization uncertainty in $R_{\mathrm{dAu}}$.

The amount of DIS data overwhelms that of the DY data. To improve upon this balance, we weight the FNAL-E772 DY data set by $w_{N}=10$. This improves the determination of the relative importance between the valence and sea quarks at intermediate values of $x$. For the NMC data set for $R_{F_{2}}^{C}$ we give a weight $w_{N}=5$ in order to better ensure a good fit for the Carbon nucleus, which is used as reference in the analysis. The NMC 96 data on the $Q^{2}$-dependence of $F_{2}^{\mathrm{Sn}} / F_{2}^{\mathrm{C}}$ is weighted by $w_{N}=10$ but only for the three lowest values of $x$ - the three upper panels in Fig. 8 below - to help constraining the gluon distribution at $x \lesssim 0.02$ via the DGLAP evolution. Finally, the few points of the BRAHMS data that we include in our analysis, are weighted by a large factor $w_{N}=40$ in order to account for the constraints this data set gives for the gluon distribution. All these weights are summarized in Table 1 above.

\section{Results}

\subsection{Final parameters}

In order to reach a well converging (well constrained) global fit, where none of the fit parameters are drifting to their limits, we are forced to reduce the total number of free parameters down to the following 15 :

\section{- Valence quark modification}

The DIS data constrain the modification $R_{V}^{A}\left(x, Q_{0}^{2}\right)$ in the $x \gtrsim 0.1$ region rather well, and altogether 8 parameters $x_{a}, y_{a}, p_{y_{a}}, x_{e}, \Delta_{e}, p_{\Delta_{e}}, b_{2}, p_{b_{2}}$ were left free.

\section{- Sea quark modification}

The DIS and DY data probe the sea quarks in the region $0.01 \lesssim x \lesssim 0.1$, and 5 parameters, $\alpha, p_{\alpha}, x_{a}, y_{a}, p_{y_{a}}$, controlling this region in $R_{S}^{A}\left(x, Q_{0}^{2}\right)$, were left free. The region $x \gtrsim 0.3$ is, however, not constrained by any present experimental data. We assume for simplicity a smooth behavior in this region, without an EMC effect.

\section{- Gluon modification}

The gluon modification $R_{G}^{A}\left(x, Q_{0}^{2}\right)$ at small $x$ is now directly constrained by the inclusive hadron production data from RHIC. Indirectly the gluons are constrained by the $Q^{2}$-evolution effects in the sea quark sector, reflected by the DIS and DY data. In spite of the new constraints, we were still able to leave only 2 parameters, $y_{a}$ and $p_{y_{a}}$ controlling the antishadowing peak height, free. We assume a similar EMC-effect for gluons as there is for valence quarks, guided by the shape of the preliminary PHENIX data for inclusive photon production in $\mathrm{Au}+\mathrm{Au}$ collisions at $p_{T} \approx 6 \mathrm{GeV}$ [33] and also by the PHENIX data for inclusive pion production 
at $\eta=0$ and $p_{T} \gtrsim 6 \mathrm{GeV}$ [15] (see Fig. 12 ahead - we have checked that indeed some sensitivity to gluon PDFs persists even at these values of $p_{T}$ ).

The global fit with these 15 parameters was then performed by minimizing the $\chi^{2}$ function defined in Eqs. (10) and (11) with the MINUIT 34 routine from the CERN Program Library. Table 2 summarizes the parameter values obtained as well as the fixed parameters. The goodness of the fit is characterized by $\chi^{2} / N=0.71$, where $\chi^{2}$ is computed with no extra weights, $w_{N}=1$, but with the optimized normalization factors $f_{N}$ included, and $N=627$ is the total number of data points. The contribution from each data set to this $\chi^{2}$ can be read off from Table 1. The corresponding nuclear modifications for selected nuclei at our initial scale $Q_{0}^{2}=1.69 \mathrm{GeV}^{2}$ are shown in Fig. 2. Table 3 shows the contribution from different types of hard processes to the unweighted $\chi^{2}$ and the comparison with our previous analysis in Ref. [3]. The $\chi^{2} / N$ obtained in earlier global analyses can be found in Table 3 of Ref. [3].

\begin{tabular}{|c|c|c|c|c|}
\hline & Param. & Valence $R_{V}^{A}$ & Sea $R_{S}^{A}$ & Gluon $R_{G}^{A}$ \\
\hline 1 & $\alpha$ & 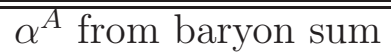 & $2.67 \times 10^{-2}$ & 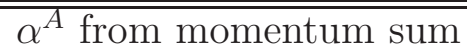 \\
\hline 2 & $p_{\alpha}$ & - & $3.47 \times 10^{-1}$ & - \\
\hline 3 & $x_{s}$ & 0.1 , fixed & 1.0, fixed & 1.0, fixed \\
\hline 4 & $p_{x_{s}}$ & 0 , fixed & 0, fixed & 0 , fixed \\
\hline 5 & $x_{a}$ & $7.37 \times 10^{-2}$ & 0.580 & 0.15 fixed \\
\hline 6 & $p_{x_{a}}$ & 0 , fixed & 0 , fixed & 0 , fixed \\
\hline 7 & $x_{e}$ & 0.751 & as valence & as valence \\
\hline 8 & $p_{x_{e}}$ & 0 , fixed & 0 , fixed & 0 , fixed \\
\hline 9 & $y_{a}$ & 1.04 & 0.997 & 1.13 \\
\hline 10 & $p_{y_{a}}$ & $1.55 \times 10^{-2}$ & $-1.51 \times 10^{-2}$ & $6.99 \times 10^{-2}$ \\
\hline 11 & $\Delta_{e}$ & 0.138 & 0 , fixed & from valence \\
\hline 12 & $p_{\Delta_{e}}$ & 0.257 & 0 , fixed & from valence \\
\hline 13 & $b_{2}$ & 13.3 & 0 , fixed & 0 , fixed \\
\hline 14 & $p_{b_{2}}$ & 0.278 & 0 , fixed & 0 , fixed \\
\hline 15 & $\beta$ & 0.3 , fixed & 0.3 , fixed & 0.3 , fixed \\
\hline 16 & $p_{\beta}$ & 0, fixed & 0, fixed & 0, fixed \\
\hline
\end{tabular}

Table 2: List of all parameters defining the modifications $R_{V}^{A}, R_{S}^{A}$ and $R_{G}^{A}$ through Eq. (3) at our initial scale $Q_{0}^{2}=1.69 \mathrm{GeV}^{2}$. The parameters $\alpha, x_{s}, x_{a}, x_{e}, y_{a}, \Delta_{e}, b_{2}$ and $\beta$ are for the reference nucleus $A=12$, and the powers $p_{i}$ define their $A$-dependence as in Eq. (4). For valence quarks and gluons, the baryon number and momentum sum rules fix the parameters $\alpha^{A}$ for each nucleus $A$ separately, in which case the powers $p_{\alpha}$ are not used. The location and height of the EMC minimum of $R_{G}^{A}$ was fixed to that of $R_{V}^{A}$. The parameters left free for the minimization procedure are shown in bold face. 


\begin{tabular}{lccc} 
Data type & Data points & $\chi_{\text {EPS08 }}^{2}$ & $\chi_{\text {EKPS }}^{2}$ \\
\hline \hline Deep Inelastic & 484 & 344.2 & 337.4 \\
Drell-Yan & 92 & 77.1 & 84.3 \\
Hadron production & 51 & 26.9 & 28.0 \\
\hline \hline Total & 627 & 448.3 & 449.6
\end{tabular}

Table 3: Contributions of various data types to the total unweighted $\chi^{2}$ in our previous work [3] (EKPS) and in this work (EPS08).

\subsection{Comparison with data}

The comparison of our results with the experimental data used in the global fit is presented in Figs. 3 - 8 for DIS, in Figs. 9 - 10 for DY, and in Figs. 11 - 12 for the RHIC data. In all figures, the open (red) symbols denote the experimental data. The error bars for the DIS and DY cases correspond to the point-to-point statistical and systematic errors added in quadrature, while for the RHIC data the statistical and systematic errors are shown separately.

In comparison with our previous work, Ref. [3], the Copper and Lithium data in Figs. 4,6 have now been added into the analysis. In Fig. 3, we see that shadowing for heavy nuclei ( $\mathrm{Sn}$ and $\mathrm{Pb}$ ) has now gotten stronger, and in Fig. 7 that we now reproduce the largest- $x$ DIS data better than before. The smallest- $x$ panel of Fig. 8 is one of the key issues in this paper and the obtained $Q^{2}$ slopes will be separately commented in Sec. 4 below. Figure 9 shows that for the DY ratios the $A$ systematics at the smallest values of $x_{2}$ have been improved: now also the Tungsten data are reproduced well. This improvement is reflected also in the large- $x_{1}$ part of the $W /$ Be ratio in Fig. 10.

The data set that plays a major role in constraining the gluon modifications in the present analysis, is the inclusive negatively-charged hadron production at forward direction $(\eta=2.2$ and $\eta=3.2)$ measured by the BRAHMS collaboration at RHIC, shown in Fig. 11. For the data sample we include in the global fit, $p_{T} \geq 2 \mathrm{GeV}$, the optimized normalization factor is close to one, $f_{N}=1.02$.

Figure 12 presents the comparison with the PHENIX and STAR measurements of inclusive pion production at midrapidity $(\eta \sim 0)$. The need of a treatment which accounts for normalization uncertainties is clearly demonstrated by this figure. Although all data sets agree within the given large uncertainties, the general trend in the STAR data is somewhat different from the PHENIX data, as can be seen in the uncorrected case $\left(f_{N}=1\right)$, shown on the left-hand side of the figure. Taking into account the normalization uncertainties as provided by the modified definition of $\chi^{2}$ in Eqs. (11), a good fit with both PHENIX and STAR data sets becomes indeed possible - see the right-hand side of Fig. 12, where the optimized normalization factors for the PHENIX data are $f_{N}=1.04$ and 1.07 and for the STAR data $f_{N}=0.90$. 


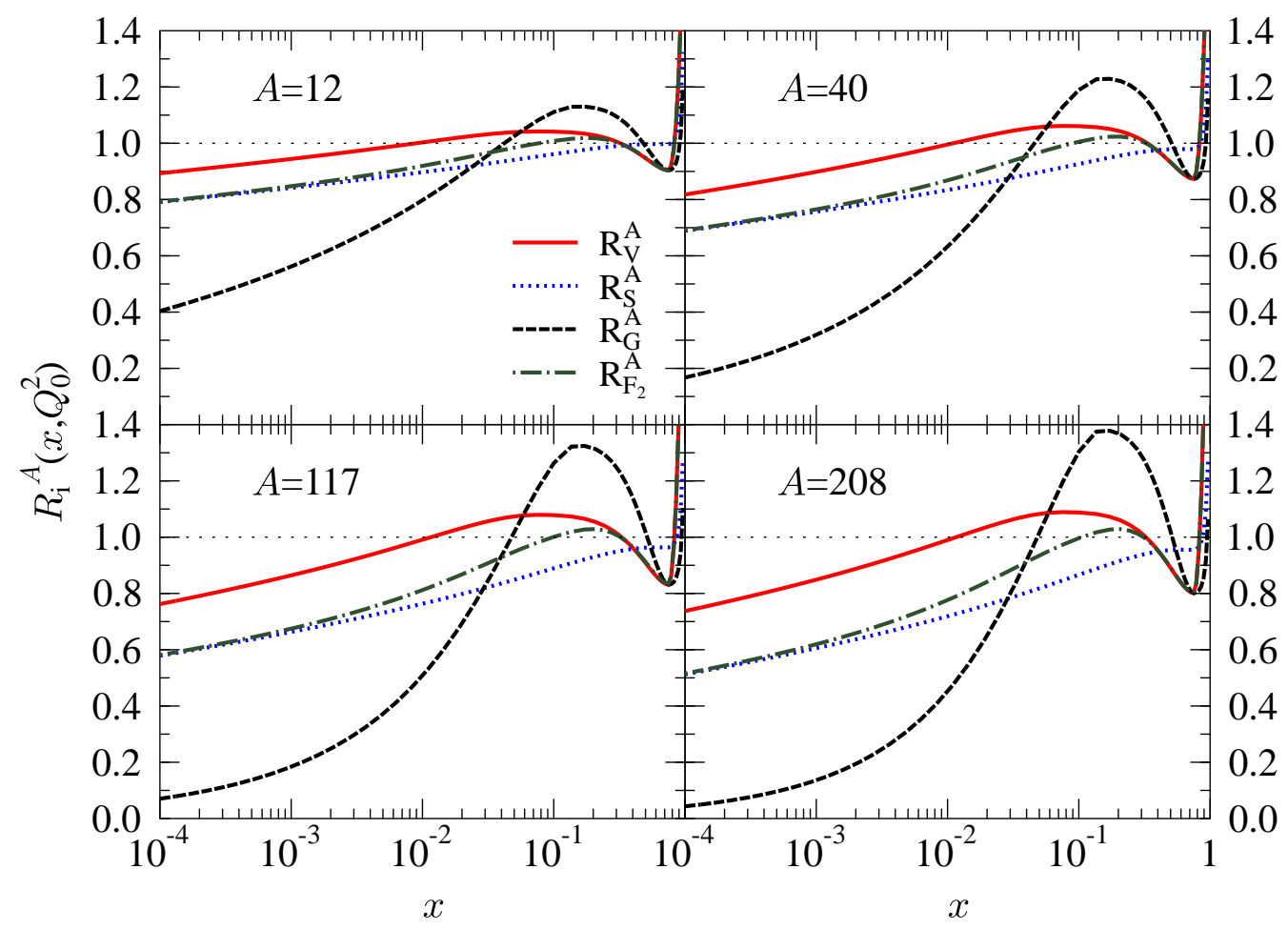

Figure 2: The nuclear modification factors $R_{V}^{A}, R_{S}^{A}$ and $R_{G}^{A}$ for $\mathrm{C}, \mathrm{Ca}, \mathrm{Sn}$, and $\mathrm{Pb}$ at $Q_{0}^{2}=1.69 \mathrm{GeV}^{2}$. The DIS ratio $R_{F_{2}}^{A}$ is shown for comparison.

\section{Discussion}

Figure 13 shows a comparison of the nuclear effects in the average valence quark, average sea quark and gluon distributions at our initial scale $Q_{0}^{2}=1.69 \mathrm{GeV}^{2}$, as obtained for a Lead nucleus in the LO DGLAP analyses here (EPS08), in HKN07 [7], in $\mathrm{nDS}$ [6], and in our previous works EKPS [3] and EKS98 [2]. The figure demonstrates the fact that while the average effects in the valence quarks and in the mid- $x$ region $(0.01 \lesssim x \lesssim 0.2)$ of the sea quarks are relatively well under control, quite large uncertainties remain in the large- $x(x \gtrsim 0.2)$ sea quark and gluon distributions. In particular, the gluon shadowing which we obtain here on the basis of the BRAHMS data, is clearly stronger than in the previous global analyses. A strong gluon shadowing has been suggested before at least in the Glauber-Gribov framework [35, 36] (see also the review [37]) and also in the context of DGLAP evolution [38, but not in a global DGLAP analysis where constraints from DIS, DY and RHIC hadron production data are simultaneously imposed.

The obtained gluon shadowing reflects a compromise between a weaker gluon shadowing suggested by the NMC 96 DIS data [27] (the first panels in Fig. 8), and a stronger effect demanded by the BRAHMS data. Regarding these constraints, we note 


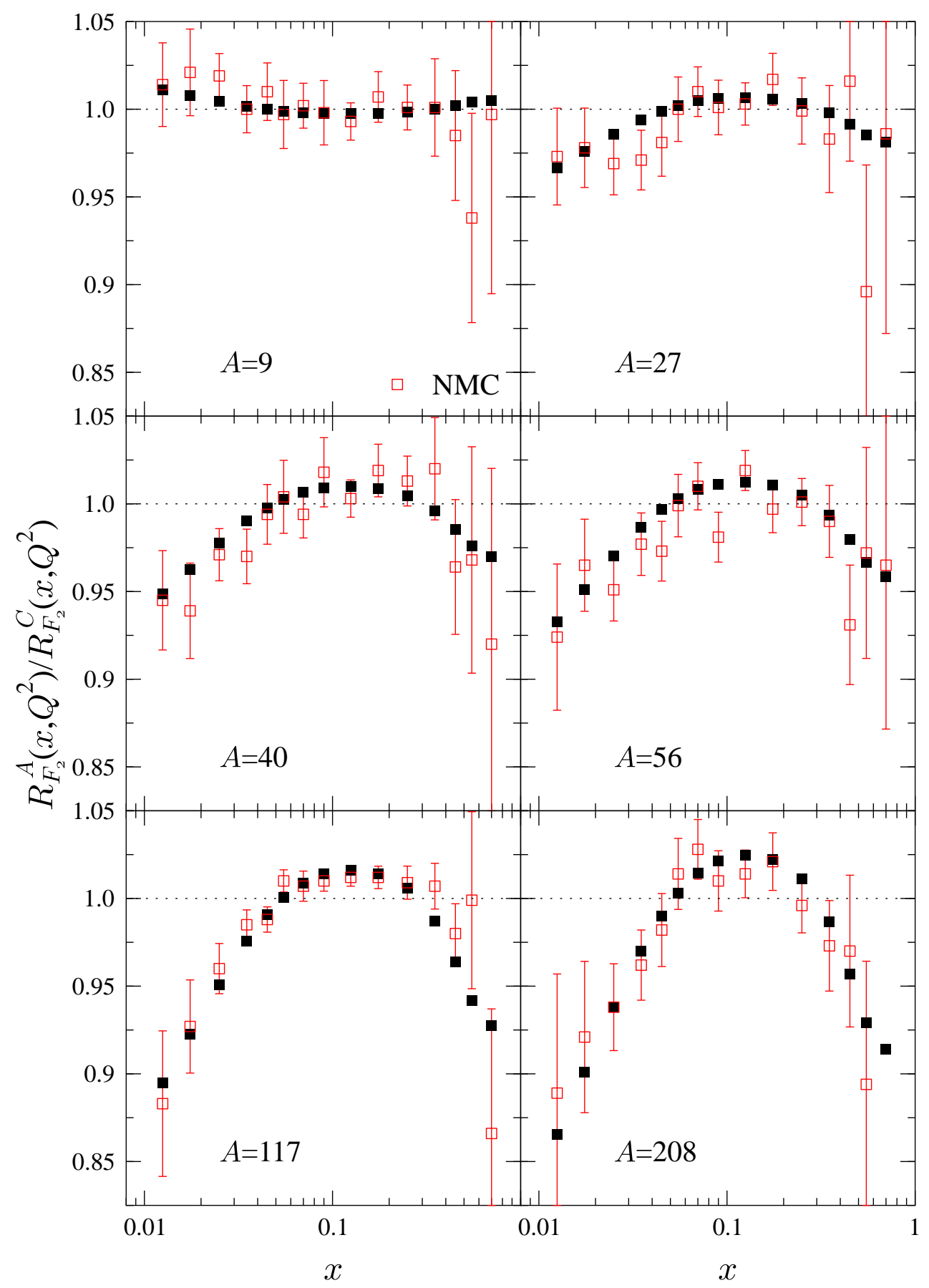

Figure 3: The computed ratio $R_{F_{2}}^{A}\left(x, Q^{2}\right)$ vs. $R_{F_{2}}^{\mathrm{C}}\left(x, Q^{2}\right)$ compared with the NMC data [28. The open symbols are the data points with statistical and systematic errors added in quadrature, the filled ones are the corresponding results from this analysis. 


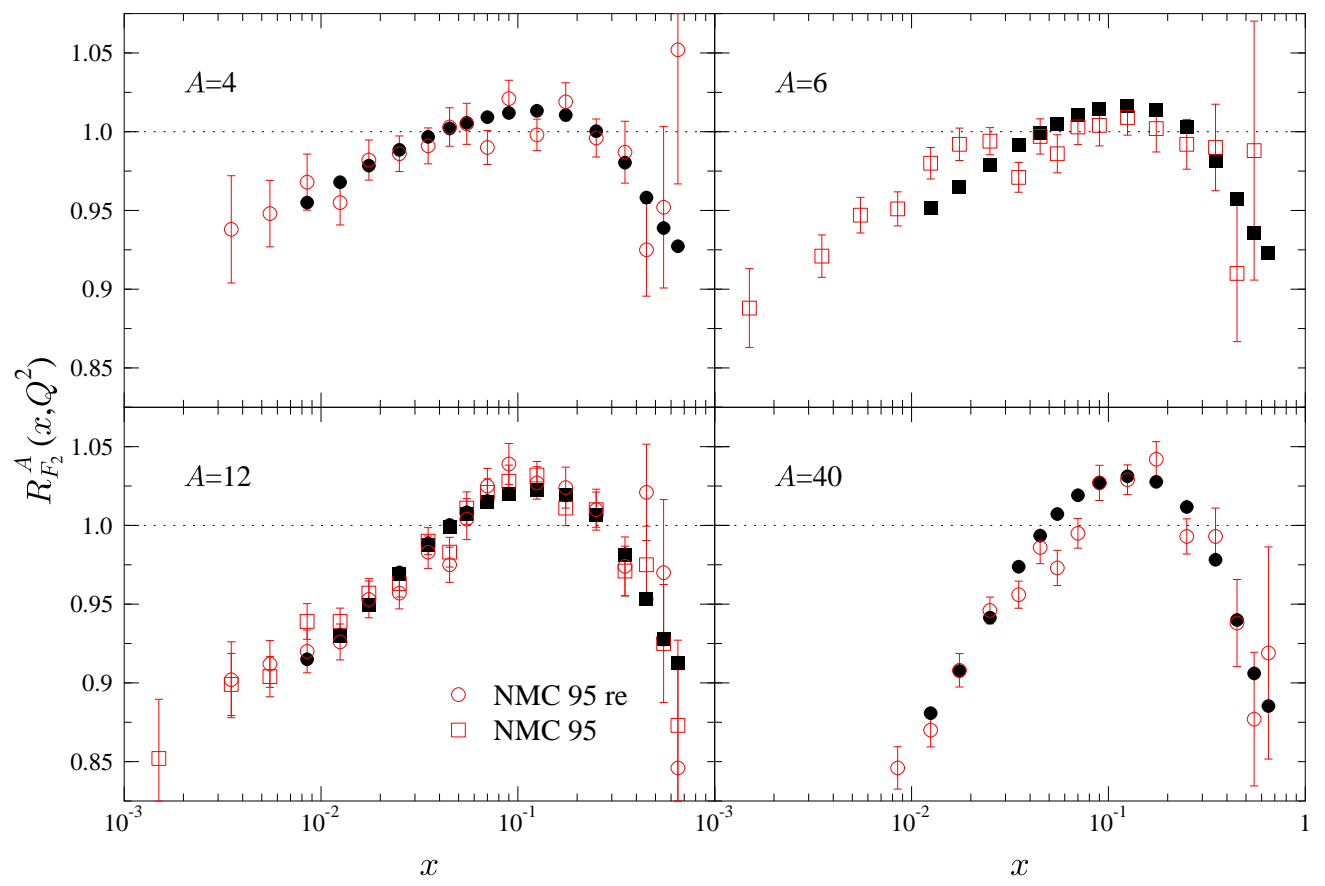

Figure 4: The calculated ratio $R_{F_{2}}^{A}\left(x, Q^{2}\right)$ compared with the NMC 95 (squares) [27] and the reanalysed NMC 95 (circles) data [26].

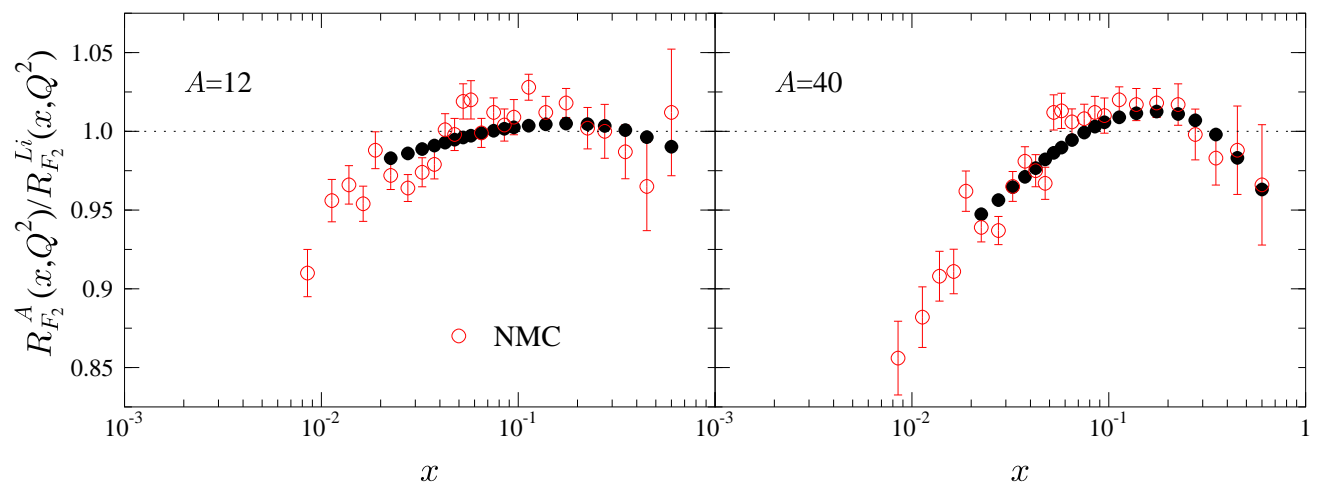

Figure 5: The computed ratio $R_{F_{2}}^{A}\left(x, Q^{2}\right)$ vs. $R_{F_{2}}^{\mathrm{L} i}\left(x, Q^{2}\right)$ (filled circles) compared with the NMC data [26] (open circles). 


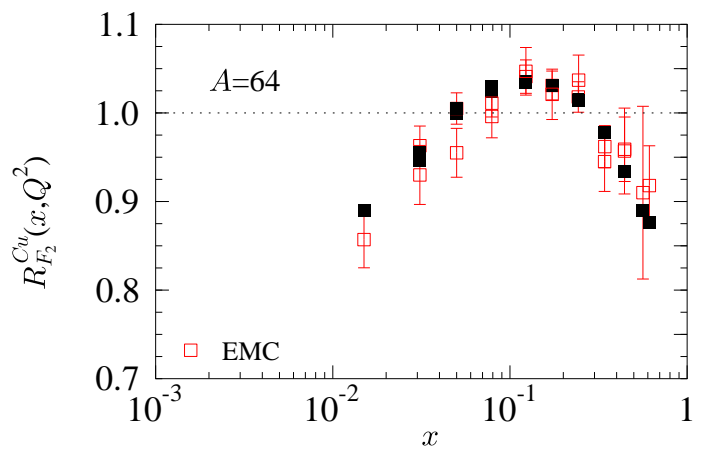

Figure 6: The calculated ratio $R_{F_{2}}^{\mathrm{Cu}}\left(x, Q^{2}\right)$ (filled squares) compared with the EMC [31] data (open squares).

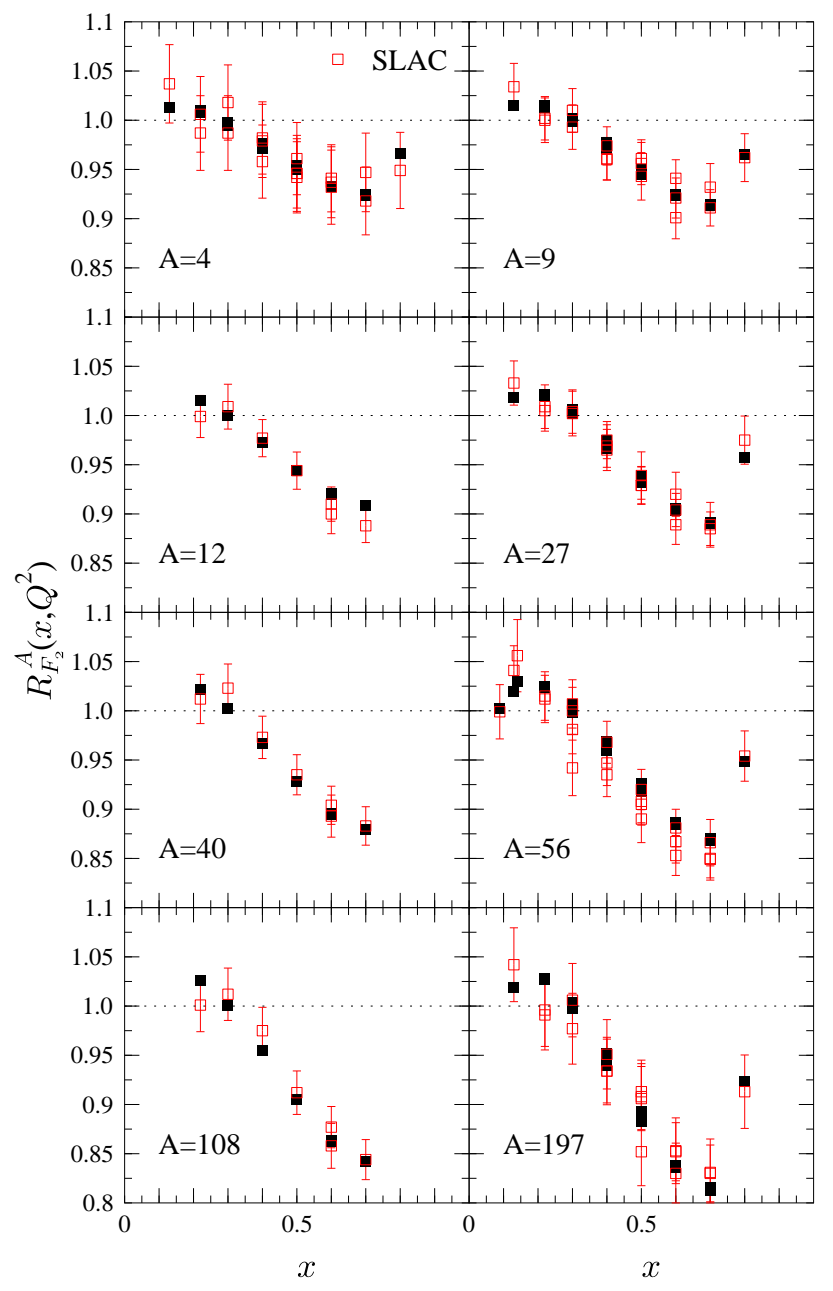

Figure 7: The calculated ratios $R_{F_{2}}^{A}\left(x, Q^{2}\right)$ (filled squares) for several nuclei compared with the SLAC data [25] (open squares). 


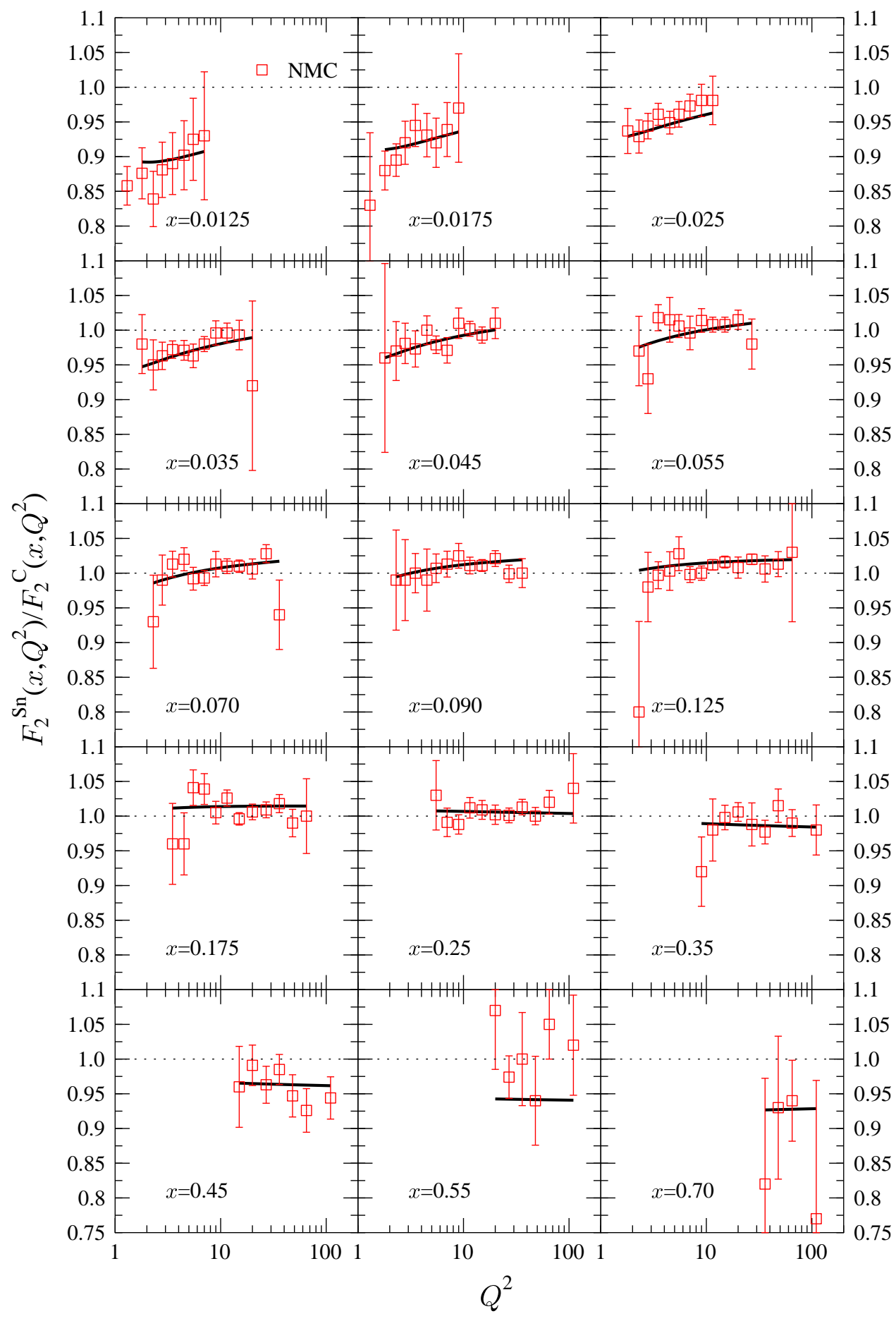

Figure 8: The calculated scale evolution (solid black lines) of the ratio $F_{2}^{\mathrm{Sn}} / F_{2}^{\mathrm{C}}$ compared with the NMC data 32 for several fixed values of $x$. 


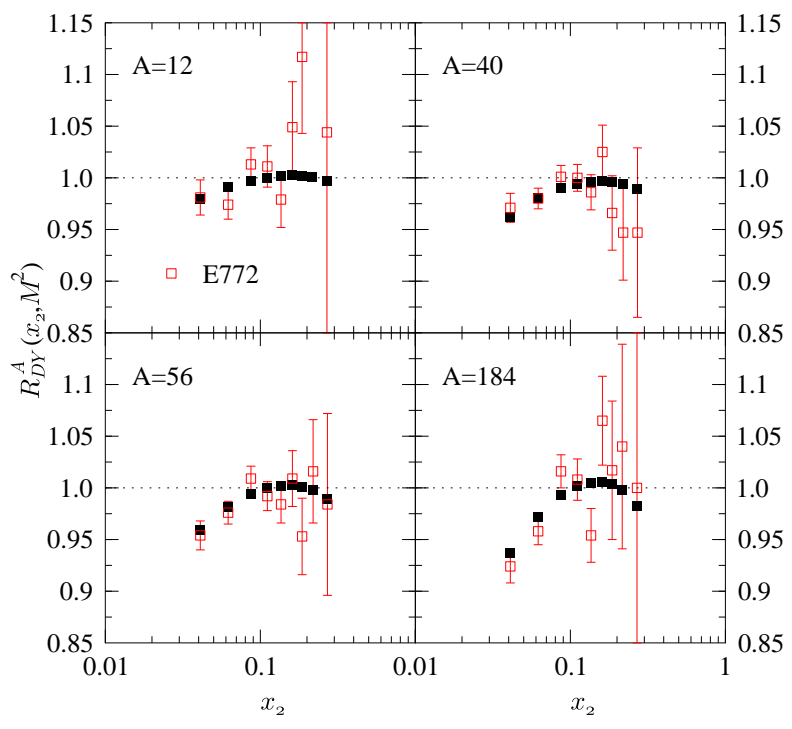

Figure 9: The computed $R_{\mathrm{DY}}^{\mathrm{A}}\left(x_{2}, M^{2}\right)$ (filled squares) as a function of $x_{2}$ compared with the E772 data [29] (open squares).

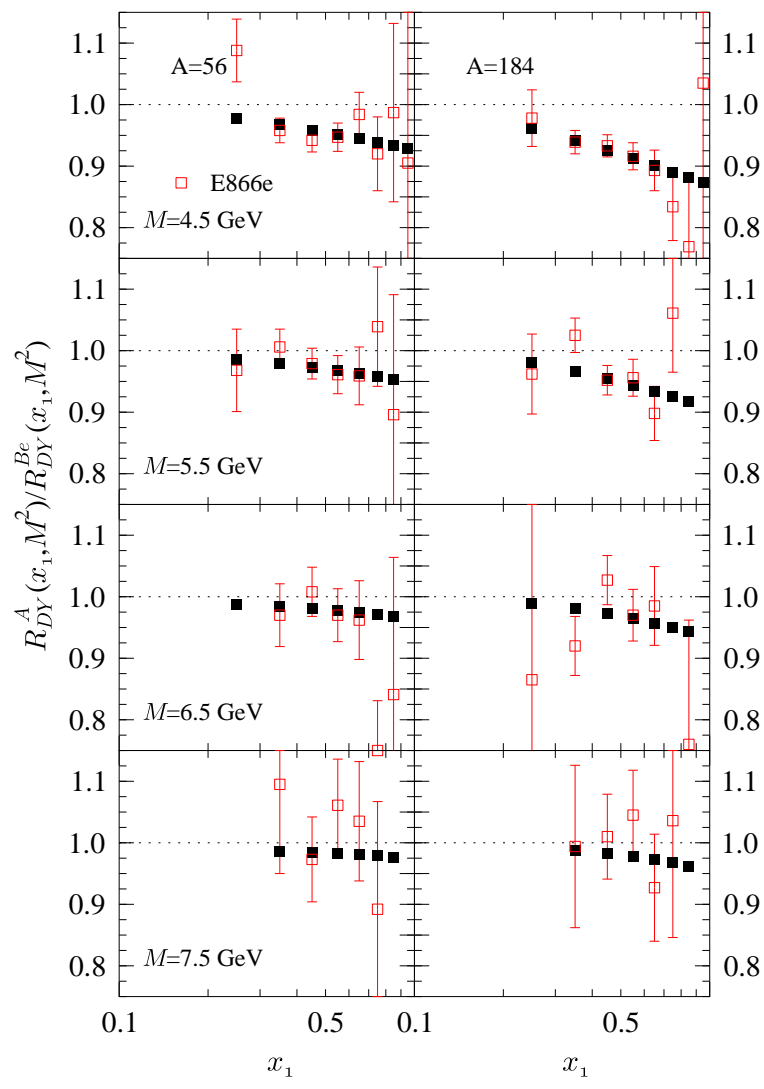

Figure 10: The computed $R_{\mathrm{DY}}^{\mathrm{A}}\left(x_{1}, M^{2}\right)$ (filled squares) as a function of $x_{1}$ compared with the E866 data [30] (open squares) at four different bins of invariant mass $M^{2}$. 

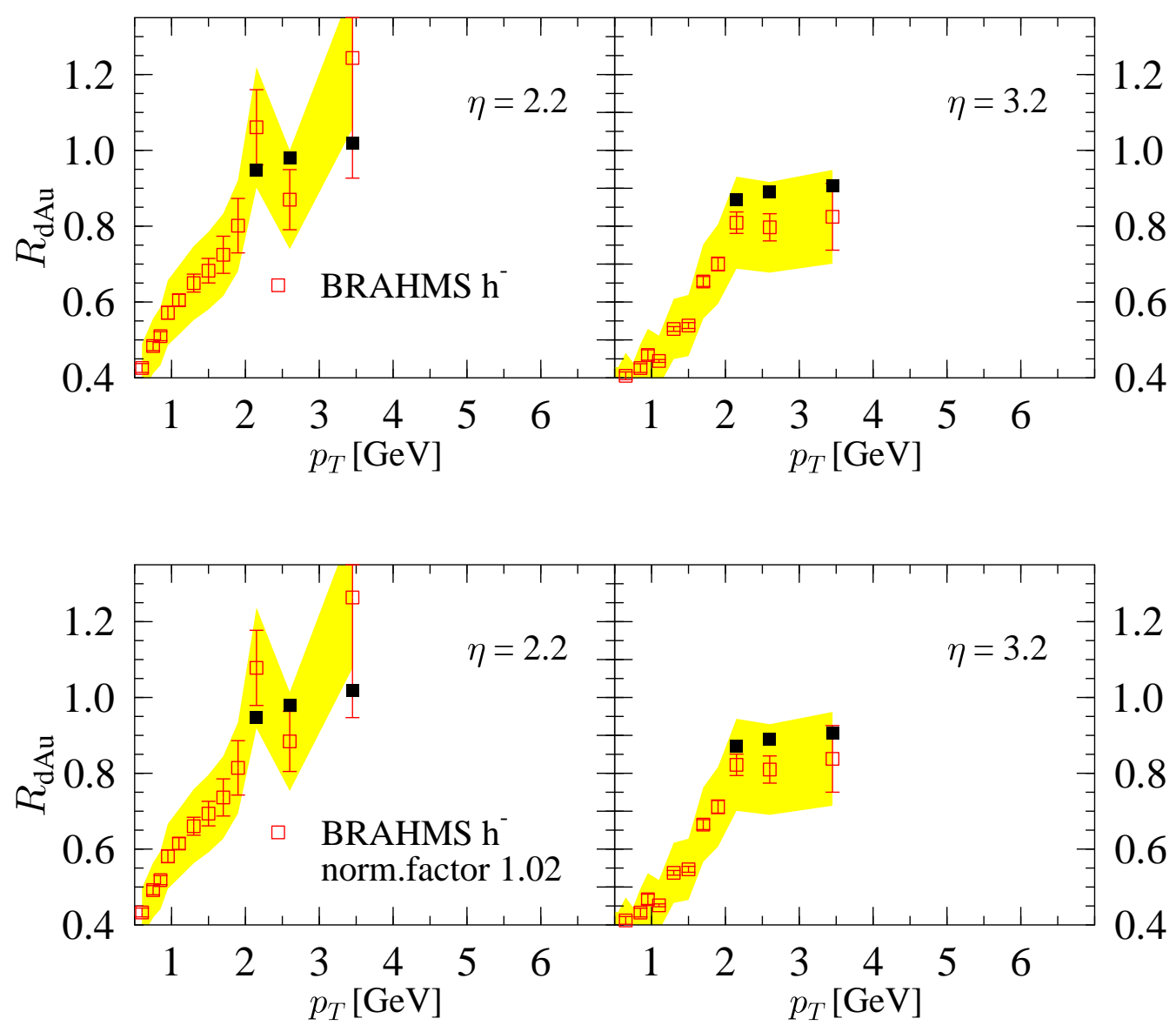

Figure 11: The computed nuclear modification ratio $R_{\mathrm{dAu}}$ at forward rapidities (filled squares) for negatively-charged hadron production, compared with the BRAHMS data [11] (open squares). The error bars are the statistical uncertainties, and the shaded bands indicate the point-to-point systematic errors. The additional overall normalization uncertainty, is $5 \%$, i.e. $\sigma_{N}^{\text {norm }}=0.05$ in Eq. (11). The upper panels show the comparison without the normalization factor $f_{N}$. In the lower panels, the data have been multiplied by the optimized value $f_{N}=1.02$. 

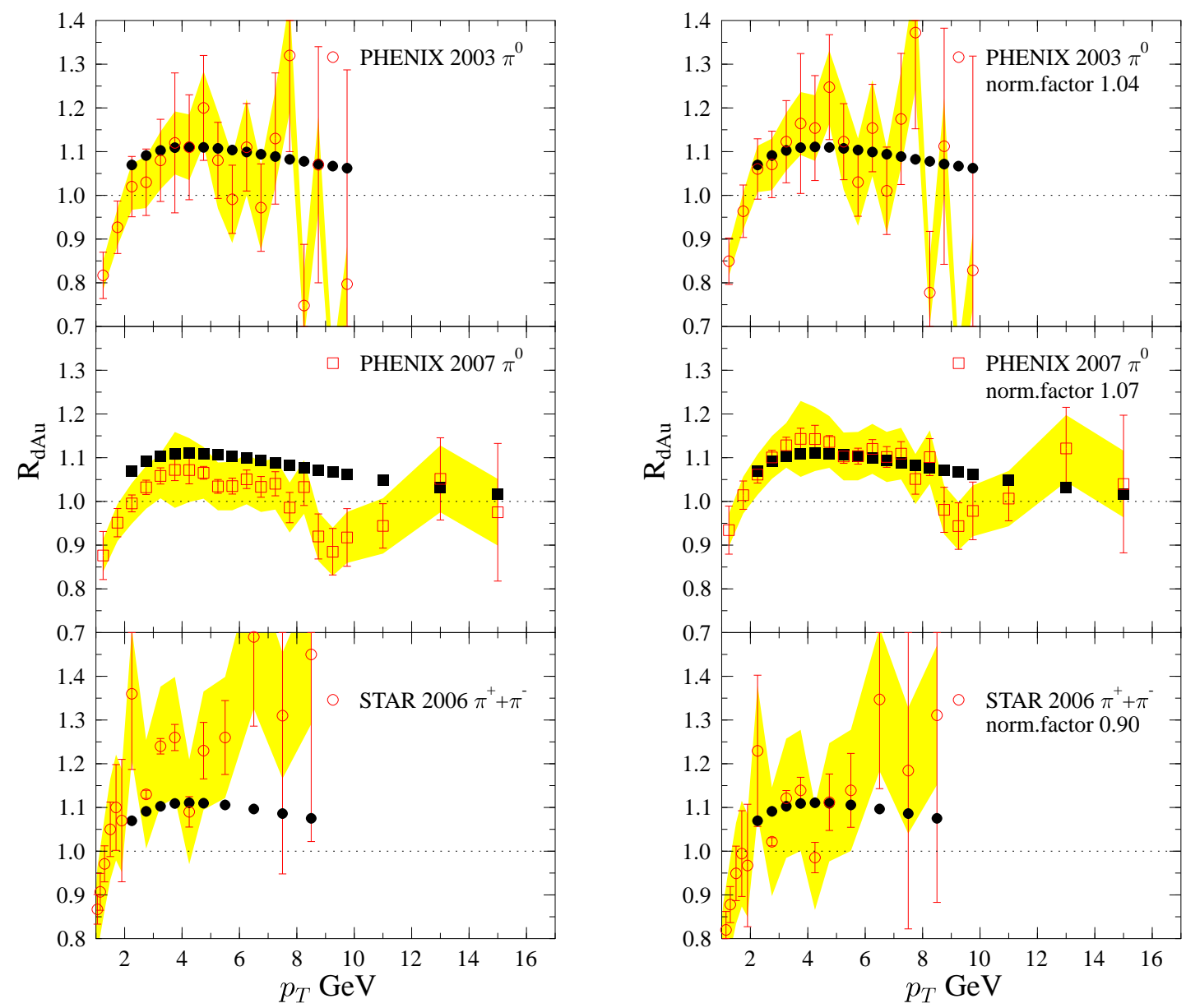

Figure 12: The computed $R_{\mathrm{dAu}}$ (filled symbols) at midrapidity $(\eta=0)$ for inclusive pion production compared with the PHENIX [14, 15] and STAR [16] data (open symbols). The error bars are the statistical uncertainties, and the shaded bands indicate the point-to-point systematic errors. The additional overall normalization uncertainties are $10 \%$ for the PHENIX data and $17 \%$ for the STAR data. The left panels show the comparison without the normalization factor $f_{N}$. In the right panels, from top to bottom, the data have been multiplied by the optimized values $f_{N}=1.04, f_{N}=1.07$ and $f_{N}=0.90$. 


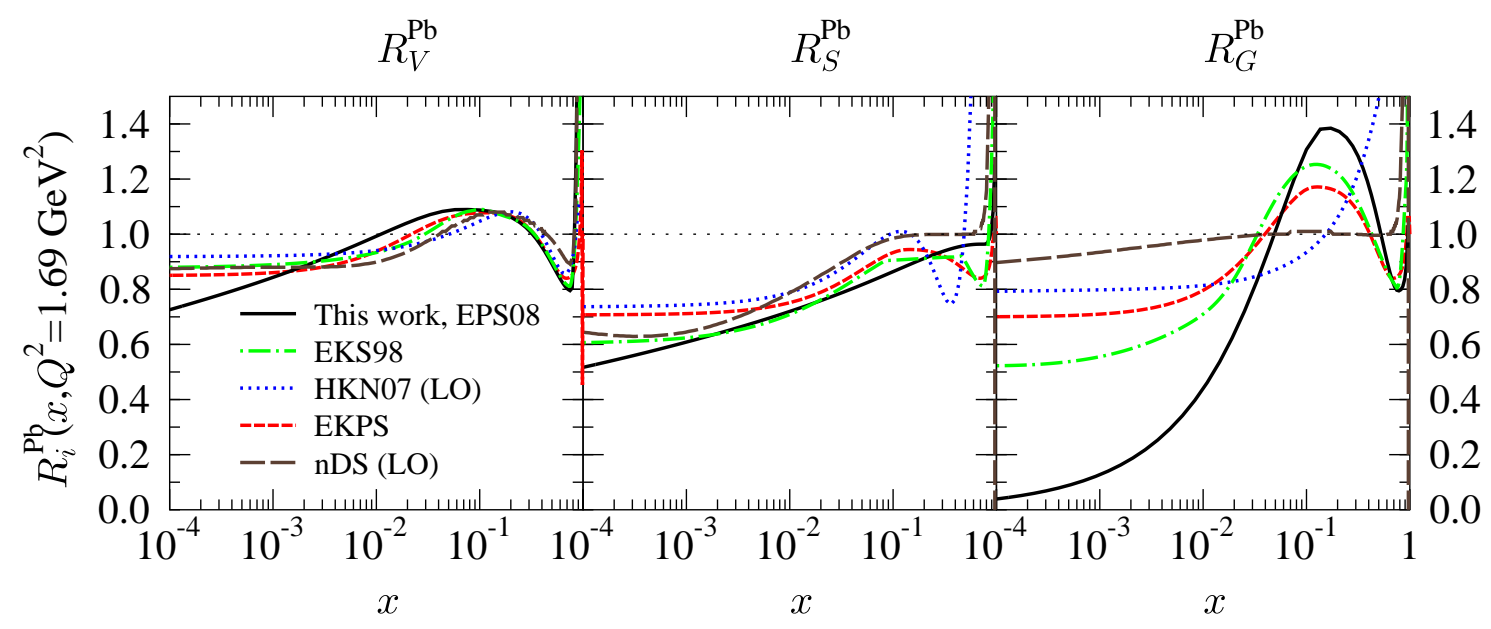

Figure 13: Comparison of the average valence and sea quark, and gluon modifications at $Q^{2}=1.69 \mathrm{GeV}^{2}$ for $\mathrm{Pb}$ nucleus from LO global DGLAP analyses EKS98 [2], HKN07 [7], nDS [6], EKPS [3] and this work EPS08.
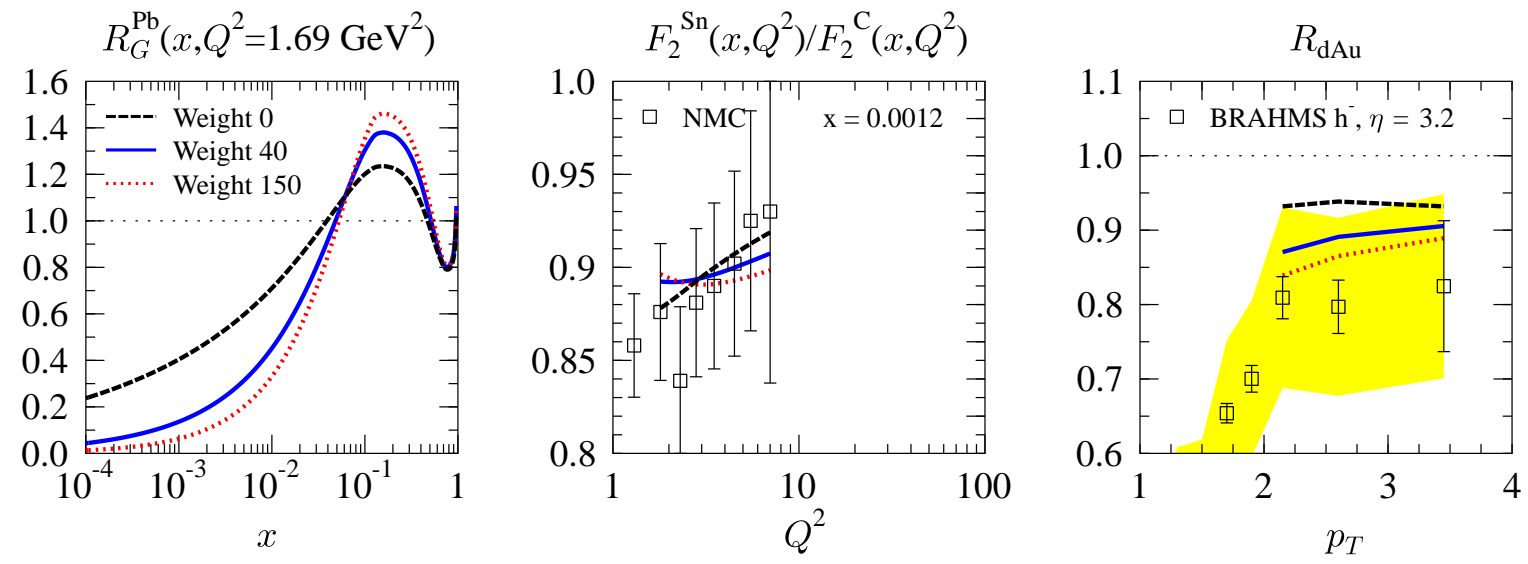

Figure 14: The correlation between the gluon shadowing (left), the $Q^{2}$ slopes of $F_{2}^{\mathrm{Sn}} / F_{2}^{\mathrm{C}}$ (middle) and the forward- $\eta R_{\mathrm{dAu}}$ (right) when weights $w_{N}=0,40$ and 150 are assigned for the BRAHMS data. In the right panel, the comparison is shown without an overall normalization factor, i.e. with $f_{N}=1$. 
the following:

First, the DIS data [27] show that the $Q^{2}$ dependence of $R_{F_{2}}$ at $x \sim 0.01$ in the region $Q^{2} \gtrsim 1 \mathrm{GeV}^{2}$ is very weak. Given this, the small- $x$ approximation of the DGLAP equations [39, 40],

$$
\frac{\partial R_{F_{2}}^{\mathrm{A}}\left(x, Q^{2}\right)}{\partial \log Q^{2}} \approx \frac{10 \alpha_{s}}{27 \pi} \frac{x g\left(2 x, Q^{2}\right)}{F_{2}^{\mathrm{D}}\left(x, Q^{2}\right)} \times\left[R_{G}^{\mathrm{A}}\left(2 x, Q^{2}\right)-R_{F_{2}}^{\mathrm{A}}\left(x, Q^{2}\right)\right],
$$

then indicates that gluon shadowing is restricted to be similar to what has been measured for $F_{2}$.

Second, using Eq. (12) above, and the fact that the $\log Q^{2}$-slope of $F_{2}^{\mathrm{Sn}} / F_{2}^{\mathrm{C}}$ measured by NMC has been observed to be positive at $x=0.125$ - see the first panel in Fig. 8 - we deduce that

$$
\left.\frac{R_{G}^{\mathrm{Sn}}\left(2 x, Q^{2}\right)}{R_{G}^{\mathrm{C}}\left(2 x, Q^{2}\right)}\right|_{x \approx 0.0125}>\left.\frac{R_{F_{2}}^{\mathrm{Sn}}\left(x, Q^{2}\right)}{R_{F_{2}}^{\mathrm{C}}\left(x, Q^{2}\right)}\right|_{x \approx 0.0125}
$$

This indicates that the $A$ dependence of gluon shadowing is weaker than that of $F_{2}$.

Third, the $A$ dependence of gluon shadowing must still be strong enough in order to reproduce the BRAHMS data. The flattening of the $Q^{2}$-slope seen in the first panel of Fig. 8 indicates that the gluon shadowing now obtained - the $A$ dependence of the gluon modifications in particular - is already so strong that it is in the brink of violating the condition (13). In this sense the gluon shadowing in the present global fit is the strongest possible one which is still in agreement with the DIS data.

Fourth, a balance between the constraints that the NMC 96 and BRAHMS data offer for the gluon shadowing, is obtained by assigning suitable relative weights, see Table 1. Since these data sets drive the fit to opposite directions, the resulting gluon shadowing obviously depends on the weights introduced. To demonstrate this sensitivity, we have repeated the analysis by varying the BRAHMS data weights as follows: By setting $w_{N}=0$, we remove this data set from the analysis. Alternatively, by assigning a very large weight, $w_{N}=150$, to the BRAHMS data, we clearly overemphasize its importance. In both cases, the smallest- $x$ NMC 96 data set weight is kept unchanged $\left(w_{N}=10\right.$, see Table 1). The overall fits obtained in these extreme cases remain very good, giving $\chi^{2} / N=0.72$ and 0.73 , correspondingly. Figure 14 (left panel) shows the resulting gluon modifications in each case, along with a comparison to the smallest- $x$ NMC 96 data (middle panel) and the BRAHMS data (right panel). The figure clearly demonstrates how adding more weight to the BRAHMS data will eventually flip the sign of the computed $Q^{2}$ slopes of $F_{2}^{\mathrm{Sn}} / F_{2}^{\mathrm{C}}$ - a phenomenon which on the basis of the systematics seen in the NMC 96 data would be an unwanted feature. With a weight factor $w_{N}=40$ for the BRAHMS data (making the effective number of the BRAHMS data points the same as in the three smallest- $x$ panels of the NMC 96 data), we reach the strongest possible gluon shadowing, and thus a fair agreement with the measured forward-rapidity $R_{\mathrm{dAu}}$, without such a sign flip.

Naively, in the RHIC hadron data, we could well expect that hadrons at fixed $\eta$ and $p_{T}$ would dominantly come from partons of higher transverse momenta, $q_{T} \sim 1.5 \ldots 2 p_{T}$ 
and the same rapidity $\eta$ [22], whose production would mainly probe the nPDFs at momentum fractions $x_{2}=\frac{q_{T}}{\sqrt{s}}\left(\mathrm{e}^{-\eta}+\mathrm{e}^{-y_{2}}\right) \approx \frac{4 p_{T}}{\sqrt{s}} \mathrm{e}^{-\eta} \sim 10^{-3}$, assuming $2 \rightarrow 2$ parton production kinematics, and taking $p_{T} \sim 2 \mathrm{GeV}$ and $y_{2} \sim \eta=3.2$. We notice, however, that the ratio $R_{\mathrm{dAu}}$ at $p_{T}=2 \mathrm{GeV}$ in Fig. 11 is considerably larger than the gluon shadowing we obtain at these values of $x$, see Fig. 13 (solid line). This is due to two reasons: First, as shown in Fig. 13 of [3], the DGLAP evolution from $Q_{0}$ to the few-GeV region increases the ratio $R_{G}^{A}$ substantially. Second, the integration over the partonic $q_{T}$ and over the unobserved parton rapidity $y_{2}$ causes a significant smearing of the $x$-range probed, especially towards larger $x$ (see also Ref. [41] and Table 1 in [10]). Thus, the ratio $R_{\mathrm{dAu}}$ at forward $\eta$ is in fact sensitive not only to nuclear gluon shadowing but also to gluon antishadowing - and antishadowing in turn amplifies when shadowing gets stronger. Therefore, even a large change in gluon shadowing induces only a moderate change in the computed ratio $R_{\mathrm{dAu}}$, and a significant gluon shadowing is required in order to reproduce the BRAHMS data at $p_{T} \geq 2 \mathrm{GeV}$.

As seen in Figs. 11] and 12, we have a good fit of the nuclear modification factor $R_{\mathrm{dAu}}$ at $p_{T} \geq 2 \mathrm{GeV}$. We cannot, however, reduce $R_{\mathrm{dAu}}$ by strengthening the gluon shadowing as much as the BRAHMS data below $2 \mathrm{GeV}$ would require without violating the DIS data constraints. This is also one of the reasons for excluding the region $p_{T}<2$ $\mathrm{GeV}$ of the RHIC data from this analysis. As explained above, we also are hesitant to push the nuclear case too far into the small- $p_{T}$ region, for we cannot reproduce the shape of the absolute $p_{T}$ spectra in $\mathrm{p}+\mathrm{p}$ collisions well enough there, and for we do not consider impact-parameter dependence of nPDFs or a more detailed centrality selection here.

As illustrated by Fig. 13, a saturation of shadowing at $x \rightarrow 0$ was assumed in previous global analyses. This assumption is now relaxed with the aim to study the strongest gluon shadowing allowed by present experimental data. It is worth emphasizing that the behavior of the nuclear corrections at the smallest values of $x\left(x \lesssim 10^{-3}\right)$ is still largely determined by the assumed shape of the fit functions in Eq. (3). This limitation is common to any global fit (for nPDFs as well as for the free proton PDFs) in those regions of phase space which are poorly or not at all constrained by the data.

\section{Conclusions}

We have improved the global analysis of nPDFs in two important ways: First, by taking the RHIC data into account in such analysis for the first time, we have extended the constrained $x$ region down to $x \gtrsim 10^{-3}$. Second, we have improved the $\chi^{2}$ minimization procedure by introducing weighting of different data sets and by explicitly accounting for the overall normalization errors quoted by the experiments.

One of the main goals of this paper is to study to what extent a strong gluon shadowing suggested by the BRAHMS data can be accommodated together with the DIS and DY data in a global analysis. We conclude that a simultaneous fit of DIS, DY and high- $p_{T}\left(p_{T} \geq 2 \mathrm{GeV}\right)$ hadron production data from RHIC at forward rapidities 
(BRAHMS negative hadrons), is indeed possible within the DGLAP framework without invoking any new suppression mechanism. Thanks to the improved treatment of data normalization errors, we obtain also a good agreement with the RHIC pion data at mid-rapidity (STAR and PHENIX). The very good quality of the global fit obtained suggests that a well-working universal set of nPDFs can be extracted in the framework of collinear factorization. Within an improved global $\chi^{2}$ analysis, and with an emphasis on the RHIC forward-rapidity data, we obtain a stronger gluon shadowing than in the previous global nPDF analyses, see Fig. 13. These are the main new results of this paper. The LO nPDF set we have obtained (EPS08), i.e. a parametrization of the $x$, $Q^{2}$ and $A$-dependent nuclear modifications relative to CTEQ6L1, is available at [17] for practical use.

As discussed above, the amount of gluon shadowing obtained depends on the weight assigned to the BRAHMS data, and equally good overall fits can be obtained also when the weights are smaller and the resulting gluon shadowing is weaker. Until more data become available to resolve this problem, the nuclear gluon distributions suffer from considerable uncertainties. To estimate the effects of these uncertainties in the hard process cross sections one computes in LO, we recommend to use the current results, EPS08, in parallel with the previous LO results EKS98 [2], nDS [6] and HKN07 [7].

Regarding inclusive hadron production in nuclear and hadronic collisions, computed here in the collinear factorization framework, we would like to emphasize that we have limited the study to the region $p_{T} \geq 2 \mathrm{GeV}$ : Within the present global analysis, we cannot reproduce the sudden drop of the ratio $R_{\mathrm{dAu}}$ measured for negative hadrons by BRAHMS at $p_{T}<2 \mathrm{GeV}$ at forward rapidities, see Fig. 11, or the very strong suppression of $R_{\mathrm{dAu}}$ measured by STAR for $\pi^{0}$ at $\eta=4$ and $p_{T}<2 \mathrm{GeV}$ [42] - a yet stronger gluon shadowing needed for this would clearly lead into a contradiction with the $\log Q^{2}$ slopes of $F_{2}^{\mathrm{Sn}} / F_{2}^{\mathrm{C}}$ measured at DIS. More detailed work on fragmentation functions, impact parameter dependence of the nPDFs as well as further developments in the fit functions is required in order to make firmer conclusions on the applicability of the DGLAP-evolved universal nPDFs in this region. Regarding the fragmentation functions, we anticipate that considering a more detailed charged separation (see e.g. [43, 44, 45]) in hadron production would tend to increase the computed $R_{\mathrm{dAu}}$ rather than decrease it. Such further complication in extracting the gluon shadowing from the BRAHMS data is, however, not considered here, since the inclusion of the charge separation becomes more reliable only in NLO.

Our next goal is to perform this analysis in NLO, as well as, when the data become finalized, include other RHIC data sets, such as photon production in $\mathrm{d}+\mathrm{Au}$ and $\mathrm{Au}+\mathrm{Au}$ from PHENIX, into the analysis. In general, any further constraints for the gluon distributions are more than welcome. For example, the ratio $R_{\mathrm{dAu}}$ for $D$ mesons to be (hopefully soon) measured at RHIC will be extremely useful, at any rapidity. The cleanest environment for the nPDFs measurements would be in the DIS experiments at eRHIC [46] and LHeC colliders now being discussed. Before the possible realization of these machines, we hope that the present study in its part demonstrates how important it would be for the correct determination of universal nPDFs to have a systematic 
proton-nucleus program also at the LHC: further constraints for nuclear gluons in the

yet unexplored regions of the $x, Q^{2}$ plane are absolutely necessary for understanding QCD parton dynamics in high-energy nuclear and hadronic collisions.

\section{Acknowledgements}

We thank the Academy of Finland, Projects 206024 and 115262, for financial support. CAS is supported by Ministerio de Educación y Ciencia of Spain under a Ramón y Cajal contract.

\section{References}

[1] K. J. Eskola, V. J. Kolhinen and P. V. Ruuskanen, Nucl. Phys. B 535 (1998) 351 arXiv:hep-ph/9802350.

[2] K. J. Eskola, V. J. Kolhinen and C. A. Salgado, Eur. Phys. J. C 9 (1999) 61 arXiv:hep-ph/9807297.

[3] K. J. Eskola, V. J. Kolhinen, H. Paukkunen and C. A. Salgado, JHEP 0705 (2007) 002 arXiv:hep-ph/0703104.

[4] M. Hirai, S. Kumano and M. Miyama, Phys. Rev. D 64 (2001) 034003 arXiv:hep-ph/0103208.

[5] M. Hirai, S. Kumano and T. H. Nagai, Phys. Rev. C 70 (2004) 044905 arXiv:hep-ph/0404093.

[6] D. de Florian and R. Sassot, Phys. Rev. D 69 (2004) 074028 arXiv:hep-ph/0311227.

[7] M. Hirai, S. Kumano and T. H. Nagai, arXiv:0709.3038 [hep-ph].

[8] I. Schienbein, J. Y. Yu, C. Keppel, J. G. Morfin, F. Olness and J. F. Owens, arXiv:0710.4897 [hep-ph].

[9] Y. L. Dokshitzer, Perturbation Theory In Quantum Sov. Phys. JETP 46 (1977) 641 [Zh. Eksp. Teor. Fiz. 73 (1977) 1216]; V. N. Gribov and L. N. Lipatov, Yad. Fiz. 15 (1972) 781 [Sov. J. Nucl. Phys. 15 (1972) 438]; V. N. Gribov and L. N. Lipatov, Yad. Fiz. 15 (1972) 1218 [Sov. J. Nucl. Phys. 15 (1972) 675]; G. Altarelli and G. Parisi, Nucl. Phys. B 126 (1977) 298.

[10] R. Vogt, Phys. Rev. C 70 (2004) 064902.

[11] I. Arsene et al. [BRAHMS Collaboration], Phys. Rev. Lett. 93 (2004) 242303 arXiv:nucl-ex/0403005. 
[12] R. Baier, A. Kovner and U. A. Wiedemann, Phys. Rev. D 68, 054009 (2003); D. Kharzeev, Y. V. Kovchegov and K. Tuchin, Phys. Rev. D 68 (2003) 094013; J. L. Albacete, N. Armesto, A. Kovner, C. A. Salgado and U. A. Wiedemann, Phys. Rev. Lett. 92, 082001 (2004).

[13] D. Stump et al., Phys. Rev. D 65 (2002) 014012 arXiv:hep-ph/0101051].

[14] S. S. Adler et al. [PHENIX Collaboration], Phys. Rev. Lett. 91 (2003) 072303 arXiv:nucl-ex/0306021.

[15] S. S. Adler et al. [PHENIX Collaboration], Phys. Rev. Lett. 98 (2007) 172302 arXiv:nucl-ex/0610036.

[16] J. Adams et al. [STAR Collaboration], Phys. Lett. B 637 (2006) 161 arXiv:nucl-ex/0601033.

[17] http://www.jyu.fi/science/laitokset/fysiikka/en/research/highenergy/urhic/nPDFs http://www-fp.usc.es/ phenom/nPDFs.

[18] J. Pumplin, D. R. Stump, J. Huston, H. L. Lai, P. Nadolsky and W. K. Tung, JHEP 0207 (2002) 012 arXiv:hep-ph/0201195.

[19] K. J. Eskola, Z. Phys. C 51 (1991) 633.

[20] P. Santorelli and E. Scrimieri, Phys. Lett. B $459 \quad$ (1999) 599 arXiv:hep-ph/9807572.

[21] B. A. Kniehl, G. Kramer and B. Potter, Nucl. Phys. B 582 (2000) 514 arXiv:hep-ph/0010289.

[22] K. J. Eskola and H. Honkanen, Nucl. Phys. A 713 (2003) 167 arXiv:hep-ph/0205048.

[23] F. Borzumati and G. Kramer, Z. Phys. C 67 (1995) 137 arXiv:hep-ph/9502280].

[24] P. Aurenche, M. Fontannaz, J. P. Guillet, B. A. Kniehl and M. Werlen, Eur. Phys. J. C 13 (2000) 347 arXiv:hep-ph/9910252.

[25] J. Gomez et al., Phys. Rev. D 49 (1994) 4348.

[26] P. Amaudruz et al. [New Muon Collaboration], Nucl. Phys. B 441 (1995) 3 arXiv:hep-ph/9503291.

[27] M. Arneodo et al. [New Muon Collaboration.], Nucl. Phys. B 441 (1995) 12 arXiv:hep-ex/9504002].

[28] M. Arneodo et al. [New Muon Collaboration], Nucl. Phys. B 481 (1996) 3. 
[29] D. M. Alde et al., Phys. Rev. Lett. 64 (1990) 2479.

[30] M. A. Vasilev et al. [FNAL E866 Collaboration], Phys. Rev. Lett. 83 (1999) 2304 arXiv:hep-ex/9906010.

[31] J. Ashman et al. [European Muon Collaboration], Z. Phys. C 57 (1993) 211.

[32] M. Arneodo et al. [New Muon Collaboration], Nucl. Phys. B 481 (1996) 23.

[33] M. J. Tannenbaum, arXiv:0707.1706 [nucl-ex].

[34] F. James and M. Roos, Comput. Phys. Commun. 10 (1975) 343.

[35] L. Frankfurt, V. Guzey and M. Strikman, Phys. Rev. D 71 (2005) 054001 arXiv:hep-ph/0303022.

[36] K. Tywoniuk, I. Arsene, L. Bravina, A. Kaidalov and E. Zabrodin, Phys. Lett. B 657 (2007) 170 arXiv:0705.1596 [hep-ph]].

[37] N. Armesto, J. Phys. G 32 (2006) R367 arXiv:hep-ph/0604108].

[38] K. J. Eskola, Nucl. Phys. B 400 (1993) 240.

[39] K. Prytz, Phys. Lett. B 311 (1993) 286.

[40] K. J. Eskola, H. Honkanen, V. J. Kolhinen and C. A. Salgado, Phys. Lett. B 532 (2002) 222 arXiv:hep-ph/0201256.

[41] V. Guzey, M. Strikman and W. Vogelsang, Phys. Lett. B 603 (2004) 173 arXiv:hep-ph/0407201.

[42] J. Adams et al. [STAR Collaboration], Phys. Rev. Lett. 97 (2006) 152302 arXiv:nucl-ex/0602011.

[43] D. de Florian, R. Sassot and M. Stratmann, Phys. Rev. D 76 (2007) 074033 arXiv:0707.1506 [hep-ph]].

[44] D. de Florian, R. Sassot and M. Stratmann, Phys. Rev. D 75 (2007) 114010 arXiv:hep-ph/0703242.

[45] S. Albino, B. A. Kniehl and G. Kramer, arXiv:0803.2768 [hep-ph].

[46] E. R. Cazaroto, F. Carvalho, V. P. Goncalves and F. S. Navarra, arXiv:0804.2507 [hep-ph]. 